\title{
Genetic architecture underlying variation in floral meristem termination in Aquilegia
}

$5{ }^{1}$ Department of Organismic and Evolutionary Biology, Harvard University, Cambridge, MA,

6 USA

$7{ }^{2}$ California State University, Sacramento, Biological Sciences, 6000 J St., Sacramento, CA,

8 USA

$9{ }^{3}$ Department of Ecology \& Marine Biology, Department of Ecology \& Marine Biology,

10 University of California, Santa Barbara, CA, USA

11 Current address: Department of Ecology and Evolutionary Biology, University of Connecticut,

12 Storrs, CT, USA

$13 *$ Corresponding author: ekramer@oeb.harvard.edu

15 ORCID:

16 Ya Min: https://orcid.org/0000-0002-7526-4516

17 Evangeline S. Ballerini: https://orcid.org/0000-0003-4810-2762

18 Scott A. Hodges: https://orcid.org/0000-0002-0401-6072

19 Elena M. Kramer: https://orcid.org/0000-0002-5757-1088

21 Main text word count:

22 Number of figures: 5

23 Number of tables: 1

24 Supplemental materials: 8 figures, 6 tables

\section{ABSTRACT}

27 Floral organs are produced by floral meristems (FMs), which harbor stem cells in their centers.

28 Since each flower only has a finite number of organs, the stem cell activity of a FM will always

29 terminate at a specific time point, a process termed floral meristem termination (FMT). Variation

30 in the timing of FMT can give rise to floral morphological diversity, but how this process is fine-

31 tuned at a developmental and evolutionary level is poorly understood. Flowers from the genus 
32 Aquilegia share identical floral organ arrangement except for stamen whorl numbers (SWN),

33 making Aquilegia a well-suited system for investigation of this process: differences in SWN

34 between species represent differences in the timing of FMT. By crossing A. canadensis and $A$.

35 brevistyla, quantitative trait locus (QTL) mapping has revealed a complex genetic architecture

36 with seven QTL. We identified potential candidate genes under each QTL and characterized

37 novel expression patterns of select candidate genes using in situ hybridization. To our

38 knowledge, this is the first attempt to dissect the genetic basis of how natural variation in the

39 timing of FMT is regulated and our results provide insight into how floral morphological

40 diversity can be generated at the meristematic level.

42 Key words: Aquilegia, floral meristem termination, stamen whorl, QTL 


\section{INTRODUCTION}

Indeterminate growth is the foundation of development in all vascular plants and is achieved by the persistent activity of stem cells in the meristems (Steeves \& Sussex, 1989). Apical meristems in the shoots and roots are highly organized structures that maintain a delicate, yet robust, balance between the production of cells that give rise to organs and the renewal of the stem cell population. In the flowering plants, when a plant enters the reproductive phase, the vegetative meristem transitions from producing leaves to floral organs. Although the overall cellular organization of the vegetative meristem and the floral meristem (FM) is highly similar, the transition to the FM identity is accompanied by a number of changes in the properties of the meristem, including changes in the rate of organ production, the patterns of primordia initiation, and an eventual loss of indeterminacy.

The loss of indeterminacy (or equivalently, the establishment of determinacy) in the FM is a well-regulated process, termed floral meristem termination (FMT), which is crucial and universal to the development of all flowers (Fig. 1). A typical flower has four types of floral organs: sepals, petals, stamens, and carpels, which are arranged from the outermost to the innermost positions of a flower (Fig. 1). Although the central stem cells stay active in the initial phase of floral organ primordia initiation, this activity will cease at a specific time point, after which all cells will be incorporated into the development of the inner most organs, the carpels (Steeves \& Sussex, 1989). The precise control of FMT is critical to ensuring that the flower has the correct number of organs, and variation in the timing of FMT is an important source of floral morphological diversity and novelty. For some species, such as Arabidopsis thaliana, FMT occurs relatively quickly, and only four whorls of floral organs are produced. In many other taxa,

66 FM activity is maintained for a more extended period; species from the Magnoliaceae,

67 Monimiaceae, Nelumbonaceae, Nymphaeaceae, Papaveraceae, and Ranunculaceae, for instance,

68 can have hundreds of spirally-arranged or whorled floral organs (Endress, 1990; Fig. 1).

69 Moreover, increased numbers of floral organs can create the raw materials for the evolution of new organ types, such as the sterile staminodes observed in Aquilegia (Ranunculaceae) or

71 Mentzelia (Loasaceae) (Walker-Larsen \& Harder, 2000). Understanding how FMT is regulated

72 in different angiosperm lineages is, therefore, interesting from both developmental and

73 evolutionary perspectives. The diversity in floral morphology of the $\sim 400,000$ angiosperm 
species is seemingly infinite, but one of the few major evolutionary trends independently observed in the transition from early-diverging angiosperms to either the core eudicot or monocot lineages is the transition from variable to stable whorl numbers in a flower (Endress, 1990), which is directly determined by the timing of FMT.
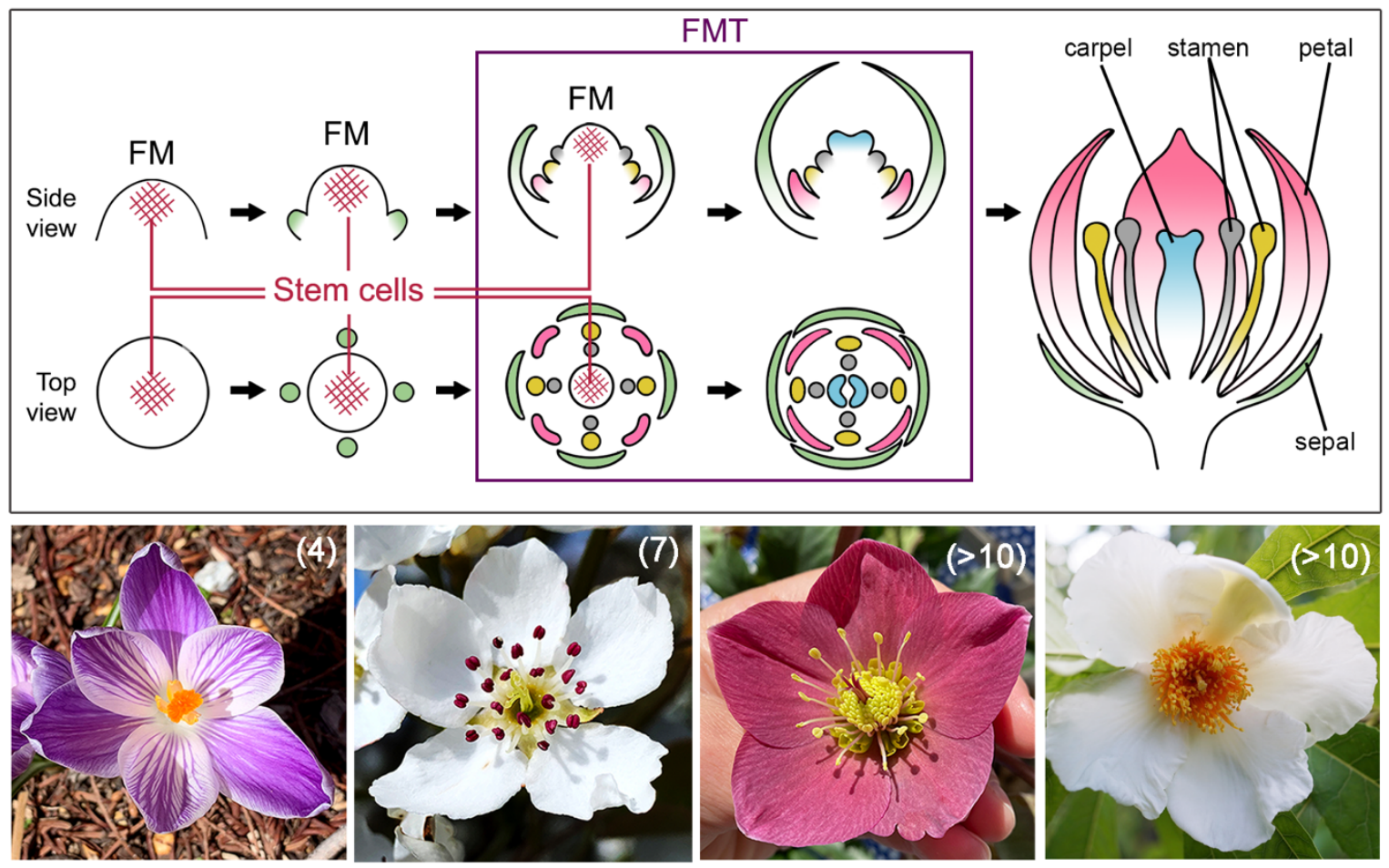

Figure 1: FMT is an important and fine-tuned developmental process that occurs in all flowers. Upper panel: diagram of floral organ initiation and FMT during flower development. Organs of the same whorl share the same colors. Lower panel: example of four flowers with different whorl numbers. From left to right: Crocus vernus 'Pickwick', Pyrus communis, Helleborus orientalis, and Franklinia alatamaha. Numbers in the parentheses indicate the number of whorls of floral organs in each flower. Photos of Crocus and Pyrus were taken by Ya Min, and photos of Helleborus and Franklinia were taken by Evangeline S. Ballerini and Jacob Suissa, respectively

Currently, we have relatively good knowledge of the genes that are responsible for maintaining and terminating stem cell activities in the A. thaliana FM, but how the timing of

81 FMT is fine-tuned at the developmental and evolutionary level is poorly understood. In the FM,

82 the maintenance of the stem cell population is achieved by a feedback loop between the

83 homeodomain protein WUSCHEL (WUS) and the CLAVATA (CLV) ligand-receptor system, in which WUS promotes central stem cell activity and induces the expression of the CLV3 peptide, while activation of the CLV signaling pathway, in turn, represses the expression of WUS (Schoof et al., 2000; Lenhard, 2003; Müller et al., 2006). In the early developing FM, the expression of 
87 the C class organ identity gene $\operatorname{AGAMOUS}(A G)$ is induced by WUS acting as a co-factor with the FM identity protein LEAFY. $A G$, in turn, specifies the identity of stamens and carpels and is also responsible for the down-regulation of WUS expression (Lenhard et al., 2001). While the broad conservation of the WUS-CLV and WUS-AG feedback loops have been demonstrated in diverse plant taxa (Nardmann \& Werr, 2006; Litt \& Kramer, 2010; Whitewoods et al., 2020), the exact mechanisms by which AG controls the precise timing of WUS down-regulation have only been investigated in A. thaliana and tomato (Solanum lycopersicum). Specifically, AG activates the expression of a $\mathrm{C} 2 \mathrm{H} 2$ zinc-finger transcription factor, $K N U C K L E S(K N U)$, which directly represses $W U S$ together with adaptor proteins from the MINI ZINC FINGER (MIF) protein family (Payne, 2004; Sun et al., 2009; Bollier et al., 2018). Accurate timing of FMT is achieved in $A$. thaliana because the activation of $K N U$ by AG takes approximately two rounds of cell divisions, during which the stamen primordia are initiated. After FMT is achieved, all of the cells remaining in the center of the FM are incorporated into the carpel primordia (Sun et al., 2009). the production of one whorl of stamens and one whorl of carpels in a flower. This begs the

102 question of whether this pathway is conserved in systems that have more than four whorls of

103 floral organs. Nonetheless, all currently established model systems (and their close relatives)

104 belong to lineages in the core eudicots or monocot grasses that exhibit no variation in their floral

105 whorl numbers, while most of the plant taxa exhibiting variation in whorls do not have the

106 genomic or genetic resources nor functional tools. Thus, they cannot provide a useful starting

107 point for the investigation of the degree of conservation in this known pathway, or how natural

108 variation in the timing of FMT is regulated in general.

109 To that end, species in the genus Aquilegia, a member of the buttercup family

110 (Ranunculaceae), are well-suited for investigating this fundamental developmental process.

111 There are approximately 70 Aquilegia species, which share low interspecific sequence

112 divergence and a high degree of interfertility due to having arisen via a recent adaptive radiation

113 (Filiault et al., 2018). In addition, a number of genetic and genomic resources have been

114 developed in in Aquilegia (Kramer, 2009; Filiault et al., 2018). All Aquilegia species (with the

115 exception of $A$. jonesii, which lacks staminodes; Munz, 1946) have the same floral organ

116 arrangement, consisting of one whorl of sepals, one whorl of petals, many whorls of stamens,

117 two whorls of staminodes (i.e., 10 in total), and one whorl of carpels (Fig. 2a). All floral organs 
118 are produced in a whorl of five, and they are arranged in 10 orthostichies (vertical rows of

119 organs), with alternate orthostichies either positioned directly above the sepals or the petals (Fig.

120 2a). Therefore, while the number of whorls of sepals, petals, staminodes (except for $A$. jonesii),

121 and carpels is consistent across the genus, the number of stamen whorls varies both within and

122 between species.

123 Aquilegia brevistyla and A. canadensis are North American sister species (Bastida et al.,

124 2010; Fior et al., 2013; Fig. 2a). Given that the number of whorls of all the non-stamen floral

125 organs is identical in these two species, the variation in what we term Stamen Whorl Number

$126(\mathrm{SWN})$ can be directly translated into variation in the timing of FMT: if the FM is terminated

127 earlier, it will have a smaller SWN compared to a flower of which the FM stays active longer.

128 Using SWN as a quantitative trait, we crossed A. brevistyla and A. canadensis and conducted

129 quantitative trait locus (QTL) mapping in the resultant F2 generation. Our results reveal a

130 complex genetic architecture with seven major QTL underlying the variation of FMT. To our

131 knowledge, this is the first study to dissect the genetic basis of how natural variation in the

132 timing of FMT is regulated, and our results highlight several potential candidate genes and

133 molecular pathways that may contribute to the regulation of FMT in Aquilegia.
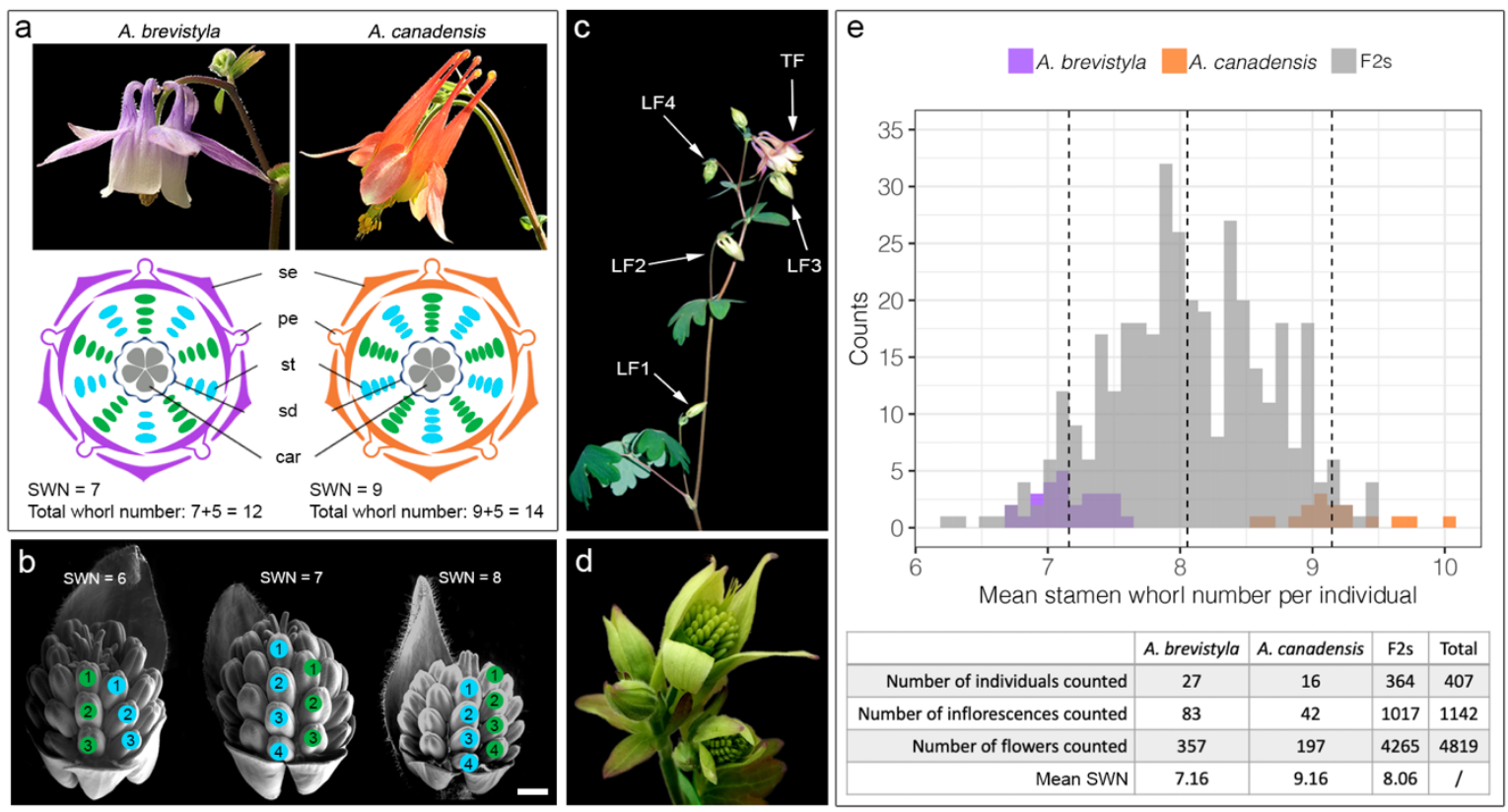

Figures 2: Phenotyping SWN in the parental and F2s populations. (a) Photos of flowers and floral diagrams of $A$. brevistyla and A. canadensis. (b) Examples of three F2 flower buds with different SWN. (c) Flowers that were sampled per inflorescence. (d) Developmental stages for which SWN were counted. (e) Histogram and summary statistics of SWN distribution in parental species and the F2s. In (a) and (b), stamen whorls positioned above the sepals are colored in blue while stamen whorls 
positioned above the petals are colored in green. Scale bar in $(b)=100 \mu \mathrm{m}$. se: sepals; pe: petals: st: stamens; sd: staminodes; car: carpels

\section{RESULTS}

\section{SWN variation in the parental species and the F2s}

We counted the SWN from 357, 197, and 4265 flowers from 27, 16, and $364 A$. brevistyla, A. canadensis, and F2 individuals, respectively (Fig. 2). The SWN per individual of the parental species did not overlap: the mean SWN of $A$. brevistyla ranges from 6.69 to 7.57; that of $A$. canadensis, from 8.54 to 10 ; and that of the F2s, which overlapped with the range of both parental species, from 6.20 to 9.50 (Fig. 2e). The mean SWN for all A. brevistyla, $A$.

143 canadensis, and F2s were 7.16, 9.16, and 8.06, respectively. Subsequently, we analyzed whether

144 the position of flowers on the inflorescence is associated with their SWN (Fig. 2c). Flower

145 position had a significant effect on the SWN for both parental species but not for the F2s (Fig.

146 S1). Given lower germination rates of interspecific hybrid seeds, seeds generated by selfing five

147 different F1 plants (all of which had the same parents) were used to get $364 \mathrm{~F} 2$ progeny that

148 reached the flowering stage ( $\mathrm{n}=53-81$ per F1). One-way ANOVA revealed that the SWN of the

149 F2s differed significantly between the F1 parents (Fig. S2). Lastly, we also analyzed the

150 variation of SWN among flowers of the same plants. Interestingly, a small portion of $A$.

151 brevistyla (7.4\%), A. canadensis (18.8\%), and F2s (6\%) showed no variance in the SWN across

152 all flowers counted within an individual, and this phenomenon was dependent on neither the

153 number of flowers counted per individual plant (Fig. S3; Pearson's correlation $=0.052, \mathrm{t}=$

$1540.98418, \mathrm{df}=345, p=0.3257$ ) nor the F1-parent-of-origin of the F2s (Fig. S3). No significant

155 correlation between the mean SWN per individual and the standard deviation of SWN per

156 individual was detected (Pearson's correlation $=0.035, \mathrm{t}=0.70438, \mathrm{df}=404, p=0.4816$ ).

\section{Floral meristem size}

To determine whether the initial FM sizes were different between the parental species, we measured the widths of FMs of the parental species at their earliest developmental stages (Fig.

1613 ). In general, the FMs of $A$. canadensis appeared to be slightly, but significantly, wider than 162 those of $A$. brevistyla throughout the early developmental stages (Fig. 3; Table S1). The average

163 FM widths of $A$. brevistyla and $A$. canadensis before the initiation of carpel primordia were 
$164174.68 \mu \mathrm{m}$ and $191.67 \mu \mathrm{m}$, respectively (Table S1). Interestingly, the temporal developmental

165 windows for significant FM size expansion seemed to be longer in A. canadensis than $A$.

166 brevistyla (Fig. 3; Table S1). The significant increase in widths of $A$. brevistyla FMs occurred

167 when there were 0 to 4 whorls of non-sepal floral organs initiating, while the significant increase

168 in the widths of $A$. canadensis FMs encompassed a larger developmental period, ranging from

169 the stages that there were 0 to 6 whorls of non-sepal floral organs initiating (Table S1).
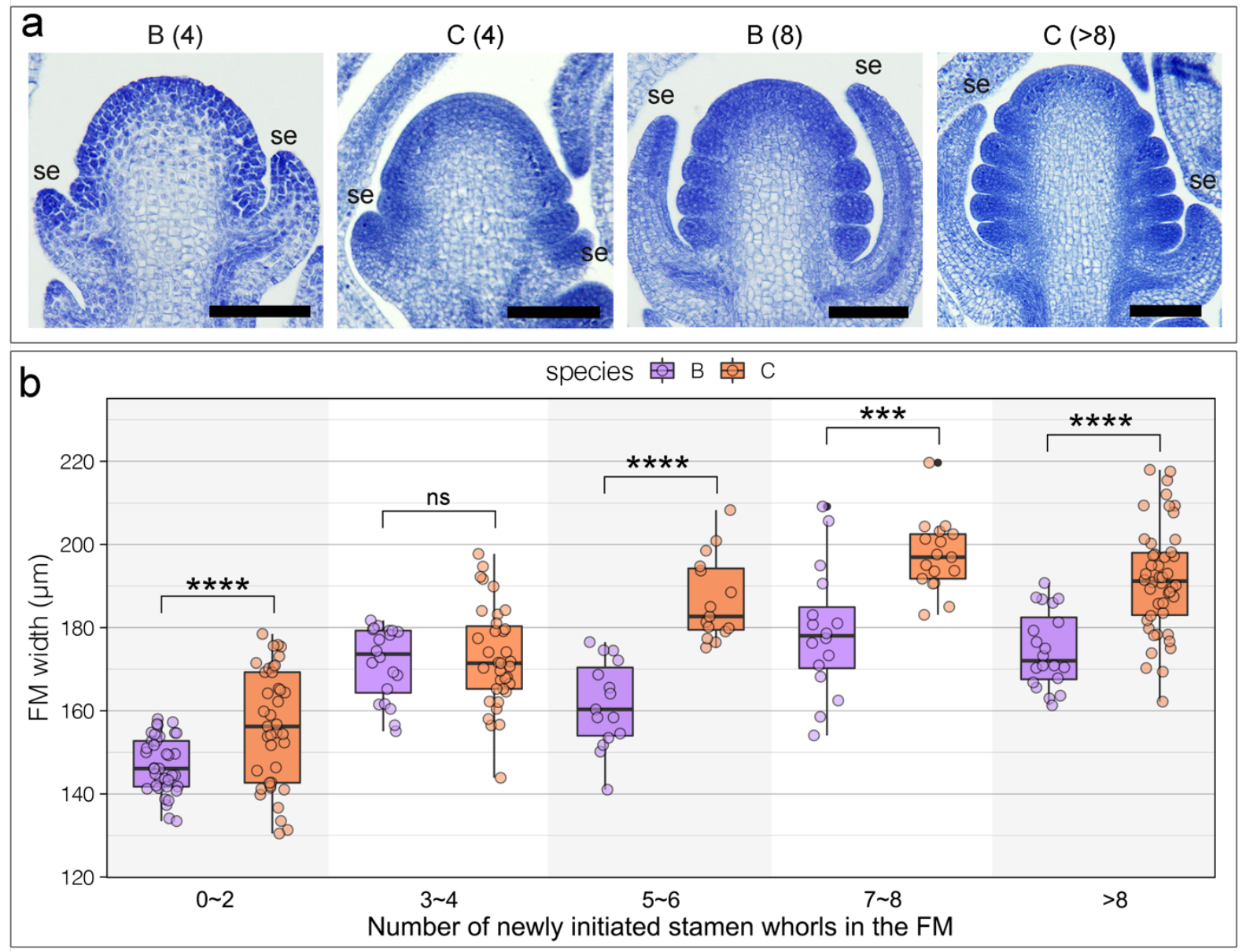

Figure 3. FM widths measurements of the parental species during the early developmental stages. (a) Examples of FM morphologies in A. brevistyla and A. canadensis at early developmental stages.

Numbers in the parenthesis indicate the number newly initiated stamen whorls in each FM. Scale bars $=100 \mu \mathrm{m}$. (b) Comparison of FM width of difference developmental stages between A. brevistyla and A. canadensis. Each data point represents a measurement of a FM width from a section. Three to six continuous sections were measured for each FM, and at least three FMs were measured for each developmental stage of each species. Comparison of FM widths of each stage between the parental species was done using Tukey's HSD. ns: not significant; ${ }^{* * *}$ : p-value $<0.001 ;{ }^{* * * *}$ : p-value $<0.0001$; se: sepal; B: A. brevistyla; C: A. canadensis.

\section{Genetic architecture underlying stamen whorl variation}


The genetic map was constructed using a total of 620 genetic markers, which fell into

173 seven linkage groups, matching the $\mathrm{n}=7$ chromosomes in the Aquilegia genome (Fig. S4). We

174 recovered seven major QTL using the mean SWN per individual as a phenotype and the F1-

175 parent-of-origin as a covariate, with one QTL on each chromosome (Fig. 4a; Table 1). The

176 difference in LOD scores between models that included or excluded the covariate are diminutive

177 on all chromosomes (ranging from -0.3 to 0.49 ; Fig. S5a), indicating no significant interaction

178 between the QTL and the covariate. While several LOD profiles from a single QTL model suggested

179 that there may be two QTL on a chromosome, a two-dimensional genome scan failed to detect evidence

180 for the presence of more than one QTL on a single chromosome (Table S2). The presence of only one

181 true QTL on chromosome 2 was further confirmed by controlling the two potential QTL: when

182 the true QTL (i.e., Q2) was controlled, the presence of the second peak also disappeared (Fig.

183 S5b). We did detect significant interactions between two pairs of QTL: Q3 and Q7, and Q1 and

184 Q6 (Fig. S5c), and thus incorporated these interactions in the full QTL model (Table 1).

185 The full QTL model had a total LOD score of 48 and explained $46.5 \%$ of the observed

186 phenotypic variation (Table 1). Q3, Q4, and Q5 exhibit larger additive effects than dominant

187 effects, while the remaining QTL have larger dominant effects on the phenotypic variation (Fig.

188 4a, S6; Table 1). The phenotypic variation explained by each QTL and the QTL interactions was

189 very similar, with Q4 and Q6 having the largest additive and dominant effects, respectively, and

190 each explained $8.8 \%$ of the phenotypic variation (Table 1 ). This suggested the genetic

191 architecture of SWN is a complex trait controlled by multiple loci, each with small effect. 

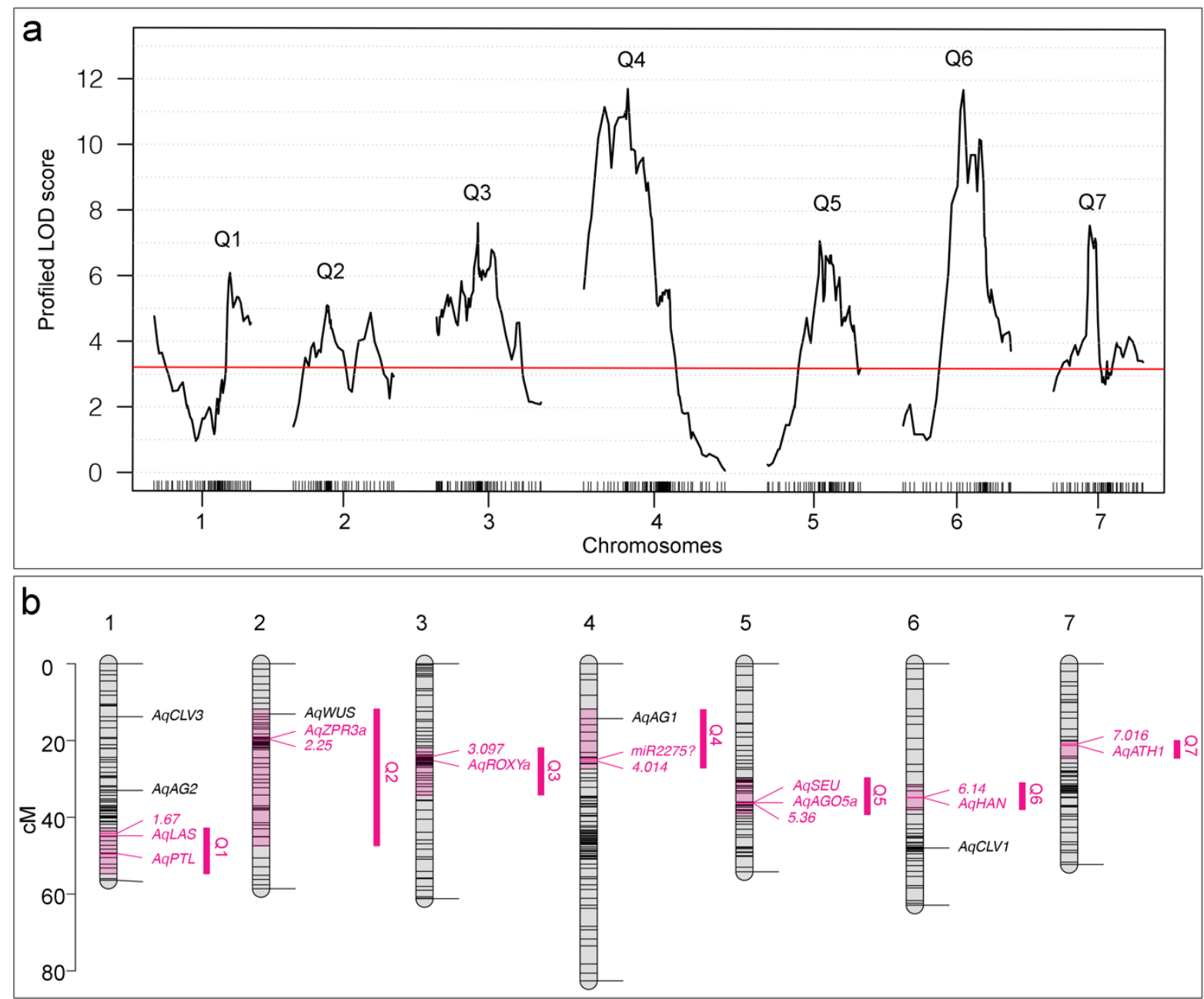

Figure 4: Genetic architecture and candidate genes. (a) LOD scores across seven chromosomes. Red line: $\alpha=0.05$ genome-wide significance cutoff based on 1000 permutations. (b) Locations of QTL interval (pink regions on the chromosomes and magenta vertical bars), candidate genes, and genetic markers. All the genetic markers were named in numeric forms (e.g., 1.67 and 2.25) and only markers with the highest LOD scores under each QTL are shown. 


\begin{tabular}{|l|l|l|l|l|l|l|}
\hline \multicolumn{7}{|l|}{ Full model result: } \\
\hline $\mathrm{y} \sim \mathrm{Q} 1+\mathrm{Q} 2+\mathrm{Q} 3+\mathrm{Q} 4+\mathrm{Q} 5+\mathrm{Q} 6+\mathrm{Q} 7+\mathrm{Q} 1: \mathrm{Q}+\mathrm{Q} 3: \mathrm{Q} 7$ \\
\hline & df & SS & MS & LOD & PVE & Pvalue(F) \\
\hline Model & 22 & 61.87 & 2.81 & 48.04 & 44.47 & 0 \\
\hline Error & 331 & 71.27 & 0.22 & & & \\
\hline Total & 353 & 133.14 & & & & \\
\hline
\end{tabular}

\section{Drop one QTL at a time ANOVA table}

\begin{tabular}{|l|l|l|l|l|l|l|l|l|l|l|}
\hline & Chr & $\begin{array}{l}\text { Position } \\
(\mathrm{cM})\end{array}$ & df & $\begin{array}{l}\text { Type } \\
\text { III SS }\end{array}$ & LOD & PVE & $\begin{array}{l}\text { F } \\
\text { value }\end{array}$ & $\begin{array}{l}\text { Pvalue } \\
(\mathrm{F})\end{array}$ & Add. & Dom. \\
\hline Q1 & 1 & 44.233 & 6 & 5.87 & 6.1 & 4.4 & 4.6 & $<0.001$ & 0.05 & -0.16 \\
\hline Q2 & 2 & 19.676 & 2 & 4.89 & 5.1 & 3.7 & 11.4 & $<0.001$ & -0.07 & 0.2 \\
\hline Q3 & 3 & 24.050 & 6 & 7.43 & 7.6 & 5.6 & 5.7 & $<0.001$ & -0.11 & -0.01 \\
\hline Q4 & 4 & 25.406 & 2 & 11.73 & 11.7 & 8.8 & 27.3 & $<0.001$ & -0.27 & 0.07 \\
\hline Q5 & 5 & 30.155 & 2 & 6.89 & 7.1 & 5.2 & 16.0 & $<0.001$ & -0.25 & 0.17 \\
\hline Q6 & 6 & 34.828 & 6 & 11.72 & 11.7 & 8.8 & 9.1 & $<0.001$ & -0.14 & -0.27 \\
\hline Q7 & 7 & 20.944 & 6 & 7.38 & 7.6 & 5.6 & 5.7 & $<0.001$ & 0 & 0.15 \\
\hline Q1 ${ }^{*}$ Q6 & 4 & 5.45 & 5.7 & 4.1 & 6.3 & $<0.001$ & & \\
\hline Q3 ${ }^{2}$ Q7 & 4 & 5.39 & 5.6 & 4.1 & 6.3 & $<0.001$ & & \\
\hline \hline
\end{tabular}

Estimated effects for QTL interactions

\begin{tabular}{|l|l|l|l|}
\hline Q1 (a)* Q6 (a): 0.01 & Q1 (d)* Q6 (a): -0.23 & Q1 (a)* Q6 (d): -0.38 & Q1 (d)* Q6 (d): 0.45 \\
\hline Q3 (a)* Q7 (a): 0.19 & Q3 (d)* Q7 (a): 0.09 & Q3 (a)* Q7 (d): -0.16 & Q3 (d)* Q7 (d): -0.24 \\
\hline
\end{tabular}

195

196

197

198

199

200

201

202

203

204

205

206

207

208

209

210 211 developmental stages (Table S3).

\section{Candidate genes}

Table 1: Summary statistics for QTL. Chr: chromosome; PVE: percent variance explained; Add./(a): additive affects; Dom./(d): dominant effects.

In order to identify potential candidate genes underlying these QTL, we examined the genomic regions defined by markers that flanked the 95\% Bayesian credible interval of each QTL, which was calculated by using the posterior distribution of $10^{\wedge} \mathrm{LOD}$ on a given chromosome. The genomic regions of Q1, Q4, Q6, and Q7 were less than $6 \mathrm{Mb}$ in size, while those of Q2, Q3, and Q5 were more than $20 \mathrm{Mb}$ (Table S3). Among all the QTL, Q6 and Q2 had the smallest $(1.5 \mathrm{Mb})$ and the largest $(36.5 \mathrm{Mb})$ intervals, containing 226 and 3242 genes, respectively (Table S3). We narrowed down the list of candidate genes by using previously published RNA-sequencing (RNAseq) data for early FMs of A. coerulea 'Kiragami', which sampled developmental stages covering the FMT window (Min \& Kramer, 2020). All QTL intervals, with the exception of Q4, had approx. 70\% of their total genes expressed in at least one of the RNAseq developmental stages; only $46.21 \%$ of the total Q4 genes were expressed in early 
bioRxiv preprint doi: https://doi.org/10.1101/2021.10.18.464884; this version posted October 19, 2021. The copyright holder for this preprint (which was not certified by peer review) is the author/funder, who has granted bioRxiv a license to display the preprint in perpetuity. It is made available under aCC-BY-NC-ND 4.0 International license.
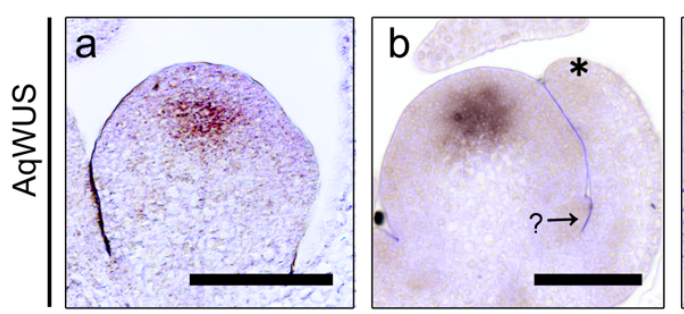

$\mathrm{br}=$ bracts; $\mathrm{c}=$ carpels; $*=$ sepals; $\rightarrow=$ petals; $\boldsymbol{\sim}=$ axillary meristems
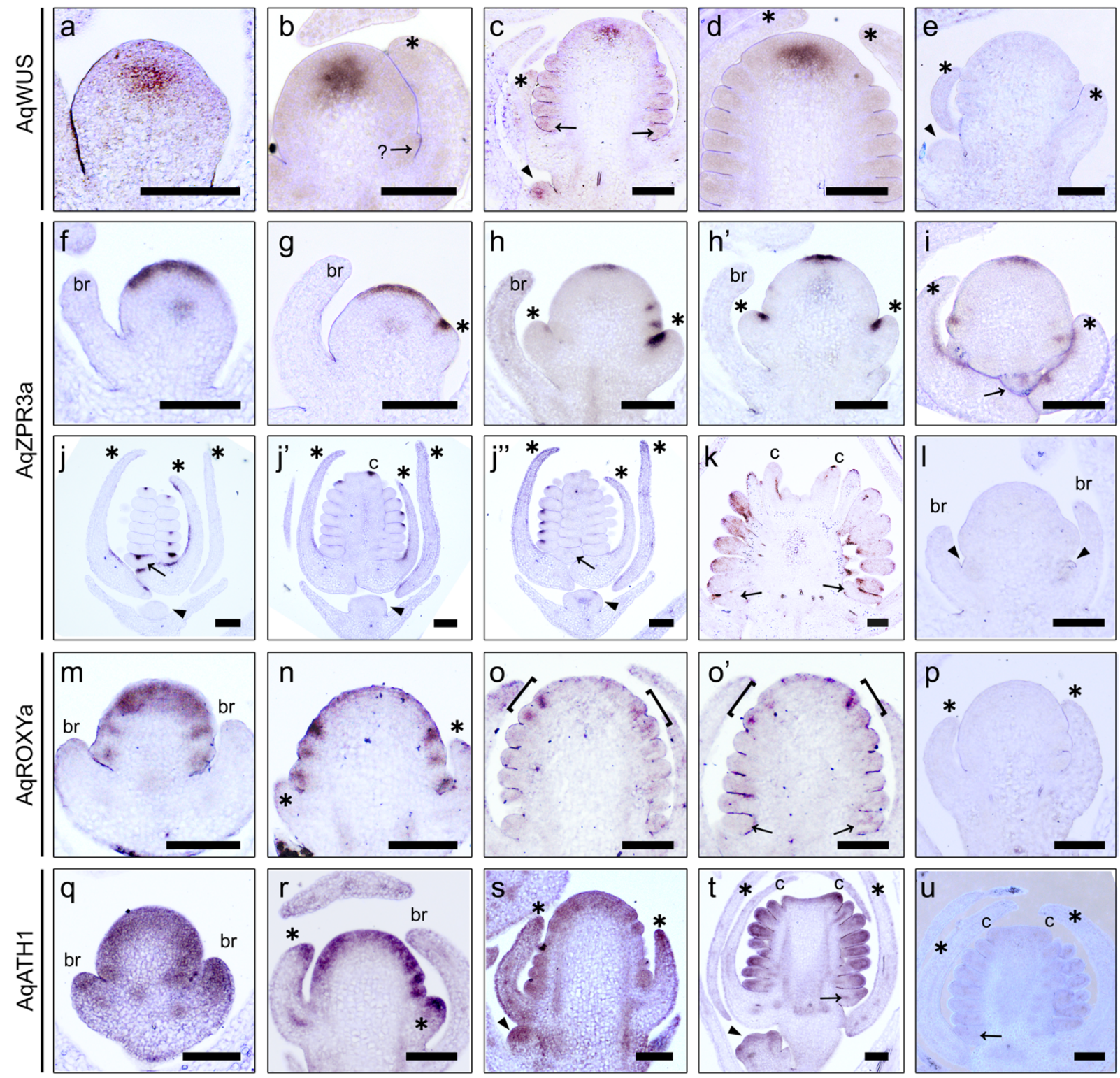

Figure 5. In situ hybridization of AqWUS and candidate genes. (a-e) Expression patterns of AqWUS (a-c) and its negative control (e). (a) A young FM that has not produced any floral organs. (b) A young FM that is in the process of initiating either a petal primordium or the outer most stamen primordia (and thus indicated by an arrow with a question mark). (c) A FM that has at least eight whorls of stamens initiated; AqWUS expression can also be seen at the axillary meristem below this FM (arrowhead). (d) A FM that has at least 11 whorls of stamens produced. (e) Sense probe on a FM with 3-4 whorls of stamens. ( $\mathrm{f}-\mathrm{I})$ Expression patterns of AqZPR3a ( $\mathrm{f}-\mathrm{k})$ and its negative control probe (I). ( $\mathrm{f}$ ) A young FM that has not produced any floral organs. (g) A young FM that has just started to produce sepal primordia (asterisk). ( $h, h^{\prime}$ ) Serial sections through the same young FM that has only produced sepal primordia (asterisks). (i) A slightly tangential section through a FM that has produced 1-2 whorls of stamens. $\left(j, j^{\prime}, j^{\prime \prime}\right)$ Serial sections through the same young floral bud that has initiated all floral organs. Expression of AqZPR3a is also e seen in the central zone of the axillary meristem in (j") (arrowhead). (k) (Figure 5 continued) A young floral bud in which all floral organs are differentiating. (I) Meristem at 
same stage as (f) hybridized with sense probe. (m-p) Expression of AqROXYa (m-o') and its negative control (p). (m) A young FM that has not produced any floral organs. ( $n$ ) A young FM that is in the process of initiating petal or the outer-most stamen primordia. (o, o') Serial sections through the same FM showing AqROXYa is only expressed on the abaxial side of the newly emerging primordia (brackets). (p) Meristem at same stage as ( $p$ ) hybridized with sense probe. ( $q-u)$ Expression of AqATH1 ( $q-t)$ and its negative control (u). (q) A young FM that has not produced any floral organs. ( $r$ ) A young FM that is in the process of initiating petal and stamen primordia. (s) A FM initiating stamen primordia and an associated axillary meristem (arrowhead). (t) A floral bud with carpel primordia just initiated and an axillary meristem, which has just initiated the sepal primordia. (u) Meristem at same stage as (t) hybridized with sense probe. All scale bars $=100 \mu \mathrm{m}$.

Subsequently, we sought to identify candidate genes under each QTL based on 1) the locations of the candidate genes relative to the location of the markers with the highest LOD scores, 2) gene expression levels during early FM developmental stages, and 3) homology to previously studied loci related to meristem function (i.e., not just restricted to FM). Because our genotyping method used $0.5 \mathrm{Mb}$ or $1 \mathrm{Mb}$ binned genomic regions as the genetic markers rather than single nucleotide markers (see details in Materials and Methods), we gave the highest priority to genes located in the region of the marker with the highest LOD scores.

Within the highest LOD bin of Q1, we identified a homolog of LATERAL SUPPRESSOR (LAS), AqLAS. LAS encodes a member of the GRAS family of putative transcriptional regulators, and mutations in the $L A S$ orthologs in A. thaliana and tomato lead to a loss of axillary meristems (Schumacher et al., 1999; Greb, 2003; Wang et al., 2014). Moreover, one additional gene within the interval that was located $3 \mathrm{Mb}$ away from $A q L A S$ also caught our attention: AqPETAL LOSS $(A q P T L) . P T L$ is a floral organ boundary gene in $A$. thaliana that controls cell proliferation in a non-cell autonomous fashion (Griffith et al., 1999; Brewer et al., 2004; Lampugnani et al., 2012). We considered $A q P T L$ interesting for two reasons. First, PTL has been shown to physically interact with and be transcriptionally regulated by $\mathrm{C} 2 \mathrm{H} 2$ transcription factor JAGGED (JAG) (Sauret-Güeto et al., 2013). In addition, silencing of $A q J A G$ led to early FM arrest in $A$. coerulea, indicating that it is an important gene in maintaining the Aquilegia FM (Min \&

231 Kramer, 2017). Second, PTL and the gene product of $H A N A B A$ TANARU $(H A N)$ interact by

232 sharing JAG as direct protein partners to regulate floral morphogenesis in $A$. thaliana (Ding et 233 al., 2015), and the homolog of $H A N$ is a candidate gene under our Q6 (Fig. 4b). edge of the interval (Fig. 4b). Since the expression of AqWUS has not been examined in situ, we analyzed its expression pattern during the early developmental stages of $A$. coerulea FM (Fig. 
5a-e). AqWUS is expressed in a small population of cells in the center of the FM from the earliest stages (Fig. 5a-e). The expression in these central zone cells persists during the initiation of floral organs and disappears when carpel primordia start to initiate. These observations are consistent with the expression of WUS orthologs in all taxa examined to date (Nardmann \& Werr, 2006; Galli \& Gallavotti, 2016), suggesting functional conservation of $A q W U S$ as well. However, the marginal position of AqWUS in Q2 makes it a less compelling candidate.

Under Q2, 8.7 Mb away from $A q W U S$ within the highest LOD interval, we identified another candidate, the gene AqLITTLE ZIPPER $3 a$ (AqZPR3a) (Fig. 4). AqZPR3a encodes a small-leucine zipper-containing protein and is the homolog of the previously identified gene ZPR3 in A. thaliana (Weits et al., 2019). Homologs of the ZPR genes have been shown to regulate leaf polarity and shoot apical meristem maintenance in A. thaliana and tomato (Wenkel et al., 2007; Kim et al., 2008; Weits et al., 2019; Xu et al., 2019), but little is known about whether they are involved in any FM-specific functions in these plant systems. In Aquilegia, $A q Z P R 3 a$ exhibits fascinating and dynamic expression patterns during early FM development (Fig. 5f-1). At the earliest stages of the FM, before floral organ primordia are initiated, concentrated expression of $A q Z P R 3 a$ is detected across the central epidermal layer of the FM, and moderate expression is found in the central zone (Fig. 5f). This strong expression in the FM epidermal layer persists until the FM has initiated several whorls of floral organs, but the width of the domain seems to contract as FM development proceeds (Fig. 5f-i). On the other hand, the expression in the central zone disappears rapidly after initiation of the sepal primordia (Fig. 5f, g, h', j')'. Strong expression of $A q Z P R 3 a$ is also detected at the adaxial boundary of all initiating floral organ primordia (Fig. 5h-j'”). However, these adaxial expression domains are restricted to the median region of the primordia, rather than the entire abaxial surface, which can be seen in 260 serial sections through the same FM in Fig. 5h, h', as well as in Fig. 5j, j', j', Moreover, strong 261 but patchy expression of $A q Z P R 3 a$ is detected in the adaxial epidermal layer of slightly older 262 lateral organs, such as the sepals (Fig. 5i, j, j'), petals (Fig. 5k), stamens (Fig. 5k) and carpels 263 (Fig. 5k). Intriguingly, expression of the ZRP genes across the epidermal layer of the early 264 meristems has never been observed in any other plant systems. Under Q3, we identified the candidate gene $A q R O X Y a$ which codes for a thioredoxin 266 superfamily protein and is a homolog of $A$. thaliana genes $R O X Y 1$ and $R O X Y 2$ (Fig. 4; Fig. S7). 267 In $A$. thaliana, $R O X Y 1$ appears to be a negative regulator of $A G$ and functions to regulate petal 
268

269

270

271

272

273

274

275

276

277

278

279

280

281

282

283

284

285

286

287

288

289

290

291

292

293

294

295

296

297

298

initiation (Xing et al., 2005; Quon et al., 2017), and ROXY2 with other members of the thioredoxin family members have been shown to regulate both floral organ development and stress responses (Xing \& Zachgo, 2008; Murmu et al., 2010; Li et al., 2019). However, the homolog of $R O X Y$ in maize has been shown to regulate shoot meristem size and phyllotaxy (Yang et al., 2015). In Aquilegia, expression of AqROXYa is detected across the FM at the earliest developmental stages (Fig. 5m), but this broad expression disappears once the primordia begin initiating. Likewise, $A q R O X Y a$ is detected in a restricted abaxial region of emerging floral organ primordia but quickly declines once they are initiated (Fig. 5m-o'). For instance, in a FM with several whorls of initiated stamen primordia, the expression of $A q R O X Y a$ is only detected in the abaxial side of the innermost two whorls of emerging organ primordia (Fig. 5o, o').

The confidence interval of Q4 spanned 3.8 Mb but only contained 176 annotated genes that were expressed in the RNAseq dataset (Table S3, S4). The only loci with homologs that are likely to function in meristems are two closely related tandem duplicates of the AGAMOUS homolog $A q A G 1$ (Fig. 4b). However, $A q A G 1$ is located at the edge of the genomic interval, quite distant from the marker with the highest LOD score (Fig. 4b). Within the $1 \mathrm{Mb}$ region $(4,800,000 . .5,800,000)$ that contained the marker with the highest LOD, there were only 40 expressed genes, eight of which are Aquilegia-specific without any annotated $A$. thaliana homologs, with most of the remaining genes annotated to be involved in plant defense and basic metabolic functions (Table S4). Since a previous study showed that microRNA2275 (miR2275) precursors and 24-PHAS loci are significantly enriched in chromosome 4 compared to other chromosomes in Aquilegia (Pokhrel et al., 2021), we also searched for potential microRNAencoding loci under Q4. MiR2275 is the primary microRNA that triggers phased, secondary, small interfering RNAs (phasiRNAs) of 24 nucleotides in length, and the production of 24-nt phasiRNAs requires both miR2275 copies and 24-PHAS loci as their targets (Liu et al., 2020). We found that the region with the highest LOD under Q4 overlaps with the genomic region with the highest density of miR2275 precursors and 24-PHAS loci, including three of the 11 annotated clusters of miR2275 precursors, and $9124-P H A S$ loci in total, comprising $31.7 \%$ of all $24-P H A S$ loci on chromosome $4(\sim 45.8 \mathrm{Mb})$ and $14.1 \%$ of such loci in entire genome ( 300 Mb) (Pokhrel et al., 2021).

For Q5, there are two genes located within the highest LOD interval that have homologs that are known to function as FM regulators: AqSEUSS (AqSEU) and AqARGONAUTE5a 
(AqAGO5a). In $A$. thaliana, $S E U$ is known to repress $A G$ to regulate FM and organ patterning (Pfluger \& Zambryski, 2004; Grigorova et al., 2011; Wynn et al., 2014), while in Aquilegia,

$301 A q A G O 5 a$ has been identified as a core hub gene associated with early FM development (Min \&

302 Kramer, 2020).

Q6 spans 1.5 Mb with 226 genes in total, 170 of which are expressed in the RNAseq dateset (Fig. 4; Table S4). Within the bin of the highest LOD score, there is one gene for which a homolog has been functionally studied: HANABA TANARU (AqHAN). HAN codes for a GATA type zinc finger transcription factor, and in A. thaliana, $H A N$ is expressed at the organ boundaries, is known to regulate WUS expression, and directly interacts with a number of key genes in FM regulation and primordia initiation (Zhao et al., 2004; Ding et al., 2015). As mentioned above, we have detected significant interaction between Q1 and Q6 (Fig. S5). We found it intriguing that HAN and PTL interact through JAG to control FM morphogenesis in $A$. thaliana (Ding et al., 2015), since their Aquilegia homologs are located within the confidence

312 interval of Q1 and Q6, respectively.

313 Lastly, Q7 also has a very narrow interval of only $2.5 \mathrm{Mb}$ in the genome with 315 genes

314 in total and 242 were expressed in the RNAseq dataset (Table S4). AqHOMEOBOX GENE 1

315 (AqATH1) is the only gene with a homolog that has been functionally studied that is also located 316 within the highest LOD interval. AqATH1 is the ortholog of gene ATH1 in A. thaliana (Fig. S8)

317 and belongs to the BELL1-like homeodomain gene family. ATH1 regulates the boundary

318 between the stem and the lateral organs, but is also involved in stem cell regulation in meristems 319 by maintaining the expression of the meristem marker gene SHOOT MERISTEMLESS via a self320 activation loop (Gómez-Mena \& Sablowski, 2008; Li et al., 2012; Cao et al., 2020). AqATH1 is 321 broadly expressed across the Aquilegia FM throughout the early developmental stages (Fig. 5qt), in all early floral organ primordia (Fig. 5r-t), and at the distal tip of the young lateral organs 323 such as the bracts (Fig. 5q), sepals (Fig. 5r, s), and petals (Fig. 5t).

\section{DISCUSSION}

\section{Aquilegia is an ideal system for studying FM regulation and termination}


330 This is despite the fact that FMT is an indispensable process in floral development and variation

331 in FMT timing is a key component of the generation of floral morphological diversity. Progress

332 in understanding the regulation and evolution of FMT is hampered due to the lack of natural

333 variation in floral organ whorl numbers in all of our currently established model systems (and

334 their close relatives), while taxa with such a variation generally lack the genomic and molecular

335 resources to investigate this question further. To this end, Aquilegia can be an ideal system for

336 studying FMT because species of Aquilegia share relatively low interspecific sequence variation

337 combined with a high degree of interfertility thanks to its recent adaptive radiation (Hodges \&

338 Arnold, 1994; Filiault et al., 2018). At the same time, they all share a consistent floral bauplan

339 that only varies in SWN (Munz, 1946), and possess a fully sequenced and well-annotated

340 genome along with RNAi-based methods for functional studies (Kramer, 2009; Filiault et al.,

341 2018). Recognizing that floral SWN is the best available quantitative trait to represent the timing

342 of FMT, we utilized a genetic cross between two sister species differing in SWN, and sought to

343 take the first step to explore the molecular basis of naturally occurring variation in the FMT

344 timing. The mean SWN of $A$. brevistyla and $A$. canadensis is 7.16 and 9.16, respectively, and

345 they do not overlap, while the mean SWN of their F2 progeny was found to encompass the entire

346 range of the parental species (Fig. 2e).

347 One question we sought to explore was whether the differences in ultimate floral size

348 between the two sister species is reflected by differences in early FM growth dynamics. By

349 analyzing developmental histological series of FMs in the parental species, we detected a subtle

350 yet significant difference in meristem diameter. Although the FMs of both species exhibit an

351 increase in width during their earliest developmental stages and then staying relatively constant

352 during the later stage, the FMs of $A$. canadensis tend to be larger at inception and grow to a

353 larger size, even before differences in SWN are evident (Fig. 3; Table S1). Overall, we observe

354 that 1) the A. canadensis FMs are larger in general, 2) have a longer developmental window to

355 increase FM width, and yet, 3) still make five stamens per whorl. There are numerous previous

356 studies showing that an increase in FM diameter is often associated with an increase in floral

357 organ number per whorl, rather than an increase in the number of whorls (e.g., Carles et al.,

358 2004; Fan et al., 2014; Chu et al., 2019). Of course, these studies typically rely on mutagenesis

359 or gene over-expression rather than natural variation. This suggests that natural variation in

360 meristem size relies on a greater degree of coordination such that meristem size changes in 
conjunction with the size of primordia inhibition fields, allowing merosity to stay constant. The current data does not allow us to distinguish between whether the $A$. canadensis FM is growing for a longer period (e.g., perhaps plastochrons are slower, allowing more mass to be accumulated between subsequent whorls) or proliferating at a faster rate. Given what we know about the role of cell division timing in influencing FMT, answering this question is important to understanding the FMT mechanism in Aquilegia. Future studies using a recently developed live imaging technique in Aquilegia (Min et al., 2021) may allow us to compare growth rates between the initiation of successive whorls in these two species and better characterize this phenomenon.

Another curious observation regarding SWN is that we observed a small portion of individuals in both parental species as well as the F2s that exhibited no variation in SWN, regardless of how many flowers were counted on the plants. In contrast, most other individuals exhibited variation in SWN within an individual plant (Fig. S3). This seems to suggest that there is variation in the robustness of this trait between different individuals. Unfortunately, the fact that there was no significant divergence in this pattern between the parent species meant that we could not map it in the current study, but we hope that examination of within-inflorescence SWN canalization in other Aquilegia species will allow the identification of suitable models and the dissection of its genetic basis.

\section{Variation in the timing of Aquilegia FMT is controlled by multiple loci of small effects}

We recovered seven major QTL that are responsible for variation in SWN, with one QTL located on each chromosome, and the percent of phenotypic variance explained by each QTL ranging from $3.7 \%$ to $8.8 \%$ (Fig. 4; Table 1). These results are comparable to previous studies in meristem-related traits of domesticated crops, particularly maize, which also revealed multiple QTL of small effects (Vlăduţu et al., 1999; Upadyayula et al., 2006; Bommert et al., 2013; maize were highly heritable, the total percentage of variance explained by all the QTL was never higher than 50\% (e.g., Bommert et al., 2013; Thompson et al., 2014, 2015), suggesting there are other loci with even smaller effects that were not picked up by the QTL mapping, which is a likely scenario for our current study as well.

We have identified the candidate genes under the QTL (Fig. 4; Table S5), and, further, uncovered novel FM expression patterns of $A q Z P R 3 a$ and $A q R O X Y a$, which were the candidate 
loci associated with Q2 and Q3, respectively (Fig. 4, 5). In A. thaliana and tomato, expression of the $Z P R$ genes is restricted to the adaxial region of lateral organs and the central zone of the shoot meristem, and the $Z P R$ genes function in both establishing organ polarity and restricting the stem cell domain in the meristems by acting as post-translational suppressors of the class III HD-ZIP abaxial identity genes by inhibiting their homodimerization (Wenkel et al., 2007; Kim et al., 2008; Weits et al., 2019; Xu et al., 2019). However, we have also observed strong expression of $A q Z P R 3 a$ in the central epidermal layer of FMs throughout their early developmental stages (Fig. 5f-i), which has not been observed in any previous studies. It will be very interesting to determine whether this expression pattern indicates a novel function or related to known ZPR functions in modulating meristem regulation. In the case of $R O X Y$ homologs in other models, expression has been found to be restricted to incipient and newly emerged organ primordia (Xing et al., 2005; Li et al., 2009b; Yang et al., 2015), but abaxialized expression such as what was found for $A q R O X Y a$ has not been observed before. In A. thaliana, ROXY1 is known to interact with $P T L$ to regulate floral primordium initiation, while in maize, a $R O X Y$ homolog controls meristem size primordia (Xing et al., 2005; Li et al., 2009b; Yang et al., 2015); either of these functions could be important for controlling FMT in Aquilegia.

The Q4 locus is of particular interest because it explains the highest relative percentage of phenotypic variation, but it is also the QTL with the fewest obvious candidate genes to investigate (Table S3, S4). Chromosome 4 of Aquilegia appears to have followed a distinct evolutionary path from the rest of the genome and displays many unique features compared to the remaining six chromosomes, including having a higher proportion of genes arrayed in tandem and segmental duplicates, more genetic polymorphism and transposable elements, lower gene density, and reduced gene expression (Filiault et al., 2018; Aköz \& Nordborg, 2019). Although the AqAG1 tandem duplication is included in the 95\% Bayesian credible interval, it may be less likely to be the causative gene compared to other genes that were located closer to the highest LOD score marker. The lack of potential candidate genes under Q4 led us to consider other factors besides protein coding genes, leading to the finding that the highest Q4 LOD interval overlaps with the region that harbors the most concentrated density of miR2275 precursors and 24-PHAS loci in the entire Aquilegia genome (Pokhrel et al., 2021). As the primary microRNA that triggers 24-nt phasiRNA, a pathway that is conserved across the angiosperms, miR2275 has been shown to be expressed in the reproductive tissues of various 
monocot and dicot lineages, particularly in developing anthers (Zhai et al., 2015; Fei et al., 2016;

424 Kakrana et al., 2018; Pokhrel et al., 2020, 2021). However, relatively little is known about 24-nt

425 phasiRNAs in general besides their functions in anthers. Overall, chromosome 4 remains an

426 enigmatic component of the Aquilegia genome, so it is intriguing that the QTL is located on this

427 structure. Certainly, it is also possible that the causal gene underlying Q4 is one of the Aquilegia-

428 specific loci that did not have a direct $A$. thaliana homolog, which equally applies to the other

429 QTL as well.

430 The current study is a key first step in identifying a promising list of candidate genes for

431 regulating natural variation in FMT. Next steps in evaluating these candidate genes will include

432 assays of gene function, conducting comparative expression analyses between $A$. canadensis and

433 A. brevistyla, and examining sequence variation and patterns of allelic differentiation between

434 populations of these species. Further areas of interest would also include exploring the potential

435 ecological consequences of variation in SWN and FMT between these species and across the

436 genus.

\section{MATERIALS AND METHODS}

\section{Plant material and growth conditions}

A. brevistyla and A. canadensis seeds were collected from wild populations in Alberta

442 (Canada) and Ithaca (NY, USA), respectively. One A. canadensis (pollen recipient) was crossed

443 with one $A$. brevistyla (pollen donor) to generate the F1 generation. Five F1s were self-fertilized

444 to generate the F2 population. All F2 seeds were stratified at $4{ }^{\circ} \mathrm{C}$ in the dark for two to four

445 weeks, germinated in wet soil, and transplanted in individual pots. All plants were vernalized at

$4464{ }^{\circ} \mathrm{C}$ for two months to induce flowering. The parental and F1 individuals were grown in the

447 greenhouse of the University of California Santa Barbara, and the F2 populations were grown in

448 the greenhouses of Harvard University. All greenhouses used the same light and temperature

449 conditions to achieve a $16 \mathrm{~h} / 8 \mathrm{~h}$ (day/night) photoperiod at $18^{\circ} \mathrm{C}$ and $13^{\circ} \mathrm{C}$.

450 Seeds of Aquilegia x coerulea 'Kiragami' were purchased from Swallowtail Garden Seeds

451 (Santa Rosa, CA, USA), germinated in wet soil, and grow under the same $18^{\circ} \mathrm{C} / 13^{\circ} \mathrm{C}$ (day/night)

452 condition as described above. Once the plants developed approx. six true leaves, they were 
transferred into vernalization conditions $\left(16 \mathrm{~h}\right.$ daylight at $6{ }^{\circ} \mathrm{C}$ and $8 \mathrm{~h}$ dark at $\left.6{ }^{\circ} \mathrm{C}\right)$ for three to four weeks, and then moved back to the regular growth conditions to promote flowering.

\section{Meristem wdith measurement}

The entire inflorescences of at least six individuals of each parental species were collected and fixed in FAA (10\% formaldehyde, 50\% ethanol, 5\% acetic acid), and stored at $4{ }^{\circ} \mathrm{C}$.

459 Samples were then dehydrated through a graded ethanol series to $100 \%$, transferred to $100 \%$

460 CitriSolv, and embedded in Paraplast Plus (Sigma-Aldrich). Embedded tissues were sectioned to

$4618 \mu \mathrm{m}$ thick ribbons with a rotary microtome, stained in $0.1 \%$ Toluidine Blue $\mathrm{O}$ solution

462 following the protocol described in Ruzin (1990), and mounted in Permount Mounting Medium

463 (Fisher Scientific). Sections were then imaged using the Axio Zoom Microscope at the Harvard

464 Center for Biological Imaging. The width of each floral meristem section was measured using

465 ImageJ. Three to six serial sections were measured for each FM, and at least three FMs were

466 measured for each developmental stage of each species. All FMs that were measured were non-

467 terminal flowers and the number of non-sepal primordia captured by the section was counted.

\section{Phenotyping}

For each plant, the SWN of the terminal flower and lateral flowers 1 to 4 from the first

471 three inflorescences were counted (Fig. 2c). If flowers of these positions in an inflorescence were

472 damaged/undeveloped, flowers at other positions were counted to achieve a total number of five

473 flowers per inflorescence. If an inflorescence produced less than five flowers, all flowers were

474 counted. SWN were counted when the flowers reached approx.1-2 cm in length (Fig. 2d)

475 because at that developmental stage, all the stamens were arranged in vertical rows, which

476 simplified counting.

\section{Genotyping}

Detailed genotyping information can be found in (Edwards et al., 2021). Briefly, the

480 DNA of the two parents that generated the cross was extracted from flash-frozen young leaves

481 using NEBNext Ultra II kit (NEB) and sequenced to $\sim 40 \mathrm{x}$ coverage as 150 bp reads on an

482 Illumina MiSeq at the Biological Nanostructures Lab in the California NanoSystem Institute at

483 UC Santa Barbara. DNA of F2s was extracted from silica dried young leaves using Qiagen 
484 DNEasy reagents and Magattract beads (Qiagen, Inc.), libraries were prepared following the 485 protocol of RipTide High Throughput Rapid DNA Library Preparation kit (iGenomX, CA, 486 USA). The F2 libraries were pooled and sequenced at the Vincent J. Coates Genomics

487 Sequencing Laboratory (UC Berkeley) using NovaSeq 6000 platform to generate 150bp paired-

488 end reads. Samples were multiplexed to generate about 1-2x coverage. All sequence data are 489 deposited in the Sequence Read Archive under BioProject ID PRJNA720109. Scripts and 490 genotype/phenotype data are available at: https://github.com/anjiballerini/can.x.brev/.

491 Sequences were aligned to the $A$. coerulea 'Goldsmith' v3.1 reference genome

492 (https://phytozome.jgi.doe.gov) using the Burrows-Wheeler aligner (Li \& Durbin, 2009) and

493 variable sites in the parents were identified using SAMtools 0.1.19 (Li et al., 2009a) with custom

494 scripts were used to identify the positions and genotypes at which the parents were homozygous

495 for different alleles. These sites were used to assign reads in the F2s as having either $A$.

496 canadensis or A. brevistyla ancestry. To determine the genotypes of the F2s, the genome

497 sequences were binned into 0.5 Mb regions with moderate to high recombination frequencies and

$4981 \mathrm{Mb}$ in regions with low or no recombination, and the frequency of reads with ancestry for each

499 F0 parent was used to determine the genotype of the bin. These bins and genotypes were used as

500 markers to construct a genetic map and conduct QTL mapping. This genotyping method has

501 been implemented in (Filiault et al., 2018; Ballerini et al., 2020; Edwards et al., 2021).

503 Mapping

After filtering out individuals and markers with more than $10 \%$ of information missing

506 of the seven chromosomes was then constructed following the protocol of the R/qtl package

507 v1.46-2 (Broman et al., 2003), with an error probability rate of 0.001 and "kosambi" map

508 function. Standard interval mapping with Haley-Knott regression (function scanone) was used

509 for the initial mapping searching for potential QTL. The best multi-QTL models are produced

510 and selected by using function stepwiseqtl, which implement penalties on different interactions

511 and drop one of the current main effects or interactions in each round of model comparison.

512 Interactions among potential QTL and between QTL and covariance were detected with a two-

513 dimensional genome scan (function scantwo). Using the estimated positions of QTL from

514 scanone, stepwiseqtl, and scantwo as the input, the positions of QTL were refined by using the 
515 function makeqtl and refineqtl, which then fit with a defined multiple-QTL model (function

516 fitqtl) with all detected interactions. F1-parent-of-origin was used as covariance in all the tested

517 mapping models. Position and effect size of QTL were estimated using drop-one-term ANOVA

518 in the best-fitting model. Chromosome diagram with candidate genes (Fig. 4) was produced by

519 using the LinkageMapView (Ouellette et al., 2018).

\section{In situ hybridization of candidate genes}

Variable regions of the genes of interest were amplified by PCR (primers in Table S6) from young inflorescence cDNA of Aquilegia x coerulea 'Kiragami'. The PCR products were

524 cloned into the $\mathrm{pCR}^{\mathrm{TM}} 4-\mathrm{TOPO}$ vectors, sequenced to confirm identity, and reverse transcribed

525 using T3 or T7 RNA polymerase and DIG RNA labeling mix (Sigma-Aldrich). Probe

526 qualification and in situ hybridization steps followed (Kramer, 2005). Slides were stained in

527 calcofluor white for 5 min before imaging, and pictures were taken using the ZEISS Axio Zoom

528 at the Harvard Center for Biological Imaging.

\section{Statistical analysis}

All statistical analyses (e.g. ANOVA, Tukey's HSD) were performed using R (version

$5321.1 .456)$.

\section{Gene trees}

Homologs of $A q R O X Y a$ and $A q A T H 1$ from various taxa were obtained by using BLAST on Phytozome (https://phytozome-next.jgi.doe.gov/). Multiple sequence alignments and

537 neighbor-joining phylogenetic trees were constructed using Geneious Prime (v2021.1.1). We did 538 not construct phylogenetic trees for other candidate genes because their homologs in A. coerulea and A. thaliana were each other's reciprocal top BLAST hits.

\section{AUTHOR CONTRIBUTIONS}

YM and EK conceived of and designed the study. EB collected seeds, crossed and

543 sequenced the parental species, and grew and pollinated the F1 individuals. ME and YM grew

544 the F2s. YM conducted all phenotyping. EB, ME, and YM prepared the libraries for sequencing

545 of the F2 individuals and constructed the genetic map. YM did all the data analysis and in situ 
546 hybridization. EK and SH provided oversight of the study. YM wrote the manuscript with input

547 from the co-authors.

\section{ACKNOWLEDGEMENT}

550 The authors would like to thank Nicole Bedford, Olivia Meyerson, and Rubén Rellán-

551 Álvarez for discussing QTL mapping analysis; Suresh Pokhrel for providing information on

552 miRNA in Aquilegia; Pierre Baduel and Rebecca Povilus for discussing data analysis strategies;

553 the graduate students at the Harvard Statistics Department who volunteered their time for

554 professional and free statistics consultation; Karl Broman for actively answering questions about

555 the R/qtl package on online forums. Funding has been provided by a Simmon's Award to YM

556 from the Harvard Center for Biological Imaging; a National Science Foundation Graduate

557 Research Fellowship under grant no. DGE1745303 to MBE; and both a NIH Ruth L. Kirschstein

558 National Research Service Award (F32GM103154) and a UC Santa Barbara Harvey Karp

559 Discovery award to ESB. Sequencing was carried out by the DNA Technologies and Expression

560 Analysis Cores at the UC Davis Genome Center, supported by NIH Shared Instrumentation

561 Grant 1S10OD010786-01 and the Biological Nanostructures Lab at UC Santa Barbara. 
Reference:

Aköz G, Nordborg M. 2019. The Aquilegia genome reveals a hybrid origin of core eudicots. Genome Biology 20: 256.

Ballerini ES, Min Y, Edwards MB, Kramer EM, Hodges SA. 2020. POPOVICH, encoding a $\mathrm{C} 2 \mathrm{H} 2$ zinc-finger transcription factor, plays a central role in the development of a key innovation, floral nectar spurs, in Aquilegia. Proceedings of the National Academy of Sciences 117: 22552-22560. Aquilegia: the biogeographical and ecological patterns of two simultaneous but contrasting radiations. Plant Systematics and Evolution 284: 171-185.

Bollier N, Sicard A, Leblond J, Latrasse D, Gonzalez N, Gévaudant F, Benhamed M, Raynaud C, Lenhard M, Chevalier C, et al. 2018. At-MINI ZINC FINGER2 and S1INHIBITOR OF MERISTEM ACTIVITY, a Conserved Missing Link in the Regulation of Floral Meristem Termination in Arabidopsis and Tomato. The Plant Cell 30: 83-100.

Bommert P, Nagasawa NS, Jackson D. 2013. Quantitative variation in maize kernel row number is controlled by the FASCIATED EAR2 locus. Nature Genetics 45: 334-337.

Brewer PB, Howles PA, Dorian K, Griffith ME, Ishida T, Kaplan-Levy RN, Kilinc A, Smyth DR. 2004. PETAL LOSS, a trihelix transcription factor gene, regulates perianth architecture in the Arabidopsis flower. Development 131: 4035-4045.

Broman KW, Wu H, Sen Ś, Churchill GA. 2003. R/qtl: QTL mapping in experimental crosses. Bioinformatics 19: 889-890.

586 Cao X, Wang J, Xiong Y, Yang H, Yang M, Ye P, Bencivenga S, Sablowski R, Jiao Y. 2020.

587 A Self-Activation Loop Maintains Meristematic Cell Fate for Branching. Current Biology 30:

588 1893-1904.e4.

589 Carles CC, Lertpiriyapong K, Reville K, Fletcher JC. 2004. The ULTRAPETALA1 gene 590 functions early in Arabidopsis development to restrict shoot apical meristem activity and acts 591 through WUSCHEL to regulate floral meristem determinacy. Genetics 167: 1893-1903.

592 Chu Y, Jang J, Huang Z, van der Knaap E. 2019. Tomato locule number and fruit size 593 controlled by natural alleles of lc and fas. Plant Direct 3.

594 Ding L, Yan S, Jiang L, Zhao W, Ning K, Zhao J, Liu X, Zhang J, Wang Q, Zhang X. 2015. 595 HANABA TARANU (HAN) Bridges Meristem and Organ Primordia Boundaries through 596 PINHEAD, JAGGED, BLADE-ON-PETIOLE2 and CYTOKININ OXIDASE 3 during Flower 597 Development in Arabidopsis (XW Deng, Ed.). PLOS Genetics 11: e1005479. 

sister species of Aquilegia (columbine). Evolution.

601 Endress PK. 1990. Patterns of floral construction in ontogeny and phylogeny. Biological 602 Journal of the Linnean Society 39: 153-175.

603 Fan C, Wu Y, Yang Q, Yang Y, Meng Q, Zhang K, Li J, Wang J, Zhou Y. 2014. A novel 604 single-nucleotide mutation in a CLAVATA3 gene homolog controls a multilocular silique trait in 605 Brassica rapa L. Molecular Plant 7: 1788-1792.

606

607

608

609

610

611

612

613

614

615

616

617

618

619

620

621

622

623

624

625

626

627

628

629

630

631

632

633

Fei Q, Yang L, Liang W, Zhang D, Meyers BC. 2016. Dynamic changes of small RNAs in rice spikelet development reveal specialized reproductive phasiRNA pathways. Journal of Experimental Botany 67: 6037-6049.

Filiault DL, Ballerini ES, Mandáková T, Aköz G, Derieg NJ, Schmutz J, Jenkins J, Grimwood J, Shu S, Hayes RD, et al. 2018. The Aquilegia genome provides insight into adaptive radiation and reveals an extraordinarily polymorphic chromosome with a unique history. eLife 7: e36426.

Fior S, Li M, Oxelman B, Viola R, Hodges SA, Ometto L, Varotto C. 2013. Spatiotemporal reconstruction of the Aquilegia rapid radiation through next-generation sequencing of rapidly evolving cpDNA regions. New Phytologist 198: 579-592.

Galli M, Gallavotti A. 2016. Expanding the Regulatory Network for Meristem Size in Plants. Trends in Genetics 32: 372-383.

Gómez-Mena C, Sablowski R. 2008. ARABIDOPSIS THALIANA HOMEOBOX GENE1 Establishes the Basal Boundaries of Shoot Organs and Controls Stem Growth. The Plant Cell 20: 2059-2072.

Greb T. 2003. Molecular analysis of the LATERAL SUPPRESSOR gene in Arabidopsis reveals a conserved control mechanism for axillary meristem formation. Genes \& Development 17: $1175-1187$.

Griffith ME, da Silva Conceição A, Smyth DR. 1999. PETAL LOSS gene regulates initiation and orientation of second whorl organs in the Arabidopsis flower. Development (Cambridge, England) 126: 5635-5644.

Grigorova B, Mara C, Hollender C, Sijacic P, Chen X, Liu Z. 2011. LEUNIG and SEUSS co-repressors regulate miR172 expression in Arabidopsis flowers. Development 138: 2451-2456.

Hodges SA, Arnold ML. 1994. Columbines: a geographically widespread species flock. Proceedings of the National Academy of Sciences 91: 5129-5132.

Kakrana A, Mathioni SM, Huang K, Hammond R, Vandivier L, Patel P, Arikit S, Shevchenko O, Harkess AE, Kingham B, et al. 2018. Plant 24-nt reproductive phasiRNAs from intramolecular duplex mRNAs in diverse monocots. Genome Research 28: 1333-1344. 

K-H, et al. 2008. HD-ZIP III Activity Is Modulated by Competitive Inhibitors via a Feedback Loop in Arabidopsis Shoot Apical Meristem Development. The Plant Cell 20: 920-933.

Kramer EM. 2005. Methods for studying the evolution of plant reproductive structures: comparative gene expression techniques. Methods in Enzymology 395: 617-636.

Kramer EM. 2009. Aquilegia : A New Model for Plant Development, Ecology, and Evolution. Annual Review of Plant Biology 60: 261-277.

641 Lampugnani ER, Kilinc A, Smyth DR. 2012. PETAL LOSS is a boundary gene that inhibits growth between developing sepals in Arabidopsis thaliana: PTL controls sepal boundaries. The Plant Journal 71: 724-735.

Lenhard M. 2003. Stem cell homeostasis in the Arabidopsis shoot meristem is regulated by intercellular movement of CLAVATA3 and its sequestration by CLAVATA1. Development 130: 3163-3173.

Lenhard M, Bohnert A, Jürgens G, Laux T. 2001. Termination of Stem Cell Maintenance in Arabidopsis Floral Meristems by Interactions between WUSCHEL and AGAMOUS. Cell 105: 805-814.

650 Li H, Durbin R. 2009. Fast and accurate short read alignment with Burrows-Wheeler transform. 651 Bioinformatics 25: 1754-1760.

652 Li H, Handsaker B, Wysoker A, Fennell T, Ruan J, Homer N, Marth G, Abecasis G, 653 Durbin R, 1000 Genome Project Data Processing Subgroup. 2009a. The Sequence

654 Alignment/Map format and SAMtools. Bioinformatics (Oxford, England) 25: 2078-2079. ROXY1, a Glutaredoxin Interacting with TGA Factors, Is Required for Petal Development in Arabidopsis thaliana. The Plant Cell 21: 429-441.

661 Li Y, Pi L, Huang H, Xu L. 2012. ATH1 and KNAT2 proteins act together in regulation of 662 plant inflorescence architecture. Journal of Experimental Botany 63: 1423-1433.

663 Litt A, Kramer EM. 2010. The ABC model and the diversification of floral organ identity. 664 Seminars in Cell \& Developmental Biology 21: 129-137.

665 Liu Y, Teng C, Xia R, Meyers BC. 2020. PhasiRNAs in Plants: Their Biogenesis, Genic 666 Sources, and Roles in Stress Responses, Development, and Reproduction. The Plant Cell 32 : $667 \quad 3059-3080$. 
Min Y, Conway SJ, Kramer EM. 2021. Quantitative live-imaging of Aquilegia floral meristems reveals distinct patterns of floral organ initiation and cell-level dynamics of floral meristem termination.

Min Y, Kramer EM. 2017. The Aquilegia JAGGED homolog promotes proliferation of adaxial cell types in both leaves and stems. New Phytologist 216: 536-548.

Min Y, Kramer EM. 2020. Transcriptome profiling and weighted gene co-expression network analysis of early floral development in Aquilegia coerulea. Scientific Reports 10: 19637.

Müller R, Borghi L, Kwiatkowska D, Laufs P, Simon R. 2006. Dynamic and Compensatory Responses of Arabidopsis Shoot and Floral Meristems to CLV3 Signaling. The Plant Cell 18:

$677 \quad 1188-1198$.

Munz PA. 1946. Aquilegia: the cultivated and wild columbines. Ithaca. NY.

Murmu J, Bush MJ, DeLong C, Li S, Xu M, Khan M, Malcolmson C, Fobert PR, Zachgo

680 S, Hepworth SR. 2010. Arabidopsis Basic Leucine-Zipper Transcription Factors TGA9 and

681 TGA10 Interact with Floral Glutaredoxins ROXY1 and ROXY2 and Are Redundantly Required 682 for Anther Development. Plant Physiology 154: 1492-1504.

Nardmann J, Werr W. 2006. The Shoot Stem Cell Niche in Angiosperms: Expression Patterns of WUS Orthologues in Rice and Maize Imply Major Modifications in the Course of Mono- and Dicot Evolution. Molecular Biology and Evolution 23: 2492-2504.

686 Ouellette LA, Reid RW, Blanchard SG, Brouwer CR. 2018. LinkageMapView—rendering high-resolution linkage and QTL maps. Bioinformatics 34: 306-307.

Payne T. 2004. KNUCKLES (KNU) encodes a $\mathrm{C} 2 \mathrm{H} 2$ zinc-finger protein that regulates development of basal pattern elements of the Arabidopsis gynoecium. Development 131: $3737-$ 3749 .

691 Pfluger J, Zambryski P. 2004. The role of SEUSS in auxin response and floral organ patterning. 692 Development 131: 4697-4707.

693 Pokhrel S, Huang K, Bélanger S, Caplan JL, Kramer EM, Meyers BC. 2020. Pre-meiotic, 694 21-nucleotide Reproductive PhasiRNAs Emerged in Seed Plants and Diversified in Flowering 695 Plants. bioRxiv: 2020.10.16.341925.

696 Pokhrel S, Huang K, Meyers BC. 2021. Conserved and non-conserved triggers of 24-nt 697 reproductive phasiRNAs in eudicots. bioRxiv: 2021.01.20.427321.

Quon T, Lampugnani ER, Smyth DR. 2017. PETAL LOSS and ROXY1 Interact to Limit 

Arabidopsis Petal Growth and Shape by Interacting with a Divergent Polarity Field (O Leyser, Ed.). PLoS Biology 11: e1001550. Population of Arabidopsis Shoot Meristems Is Maintained by a Regulatory Loop between the CLAVATA and WUSCHEL Genes. Cell 100: 635-644.

Schumacher K, Schmitt T, Rossberg M, Schmitz G, Theres K. 1999. The Lateral suppressor (Ls) gene of tomato encodes a new member of the VHIID protein family. Proceedings of the National Academy of Sciences 96: 290-295.

Steeves TA, Sussex IM. 1989. Patterns in Plant Development. Cambridge University Press.

Sun B, Xu Y, Ng K-H, Ito T. 2009. A timing mechanism for stem cell maintenance and differentiation in the Arabidopsis floral meristem. Genes \& Development 23: 1791-1804. MJ, Muehlbauer GJ. 2014. Genetic Control of Maize Shoot Apical Meristem Architecture. G3 Genes|Genomes|Genetics 4: 1327-1337. Relationship to Plant Morphology. G3 Genes|Genomes|Genetics 5: 819-827. Vegetative to Generative Structures. Genetics 153: 993-1007.

Walker-Larsen J, Harder LD. 2000. The evolution of staminodes in angiosperms: patterns of stamen reduction, loss, and functional re-invention. American Journal of Botany 87: 1367-1384. The Plant Cell 26: 2068-2079.

Weits DA, Kunkowska AB, Kamps NCW, Portz KMS, Packbier NK, Nemec Venza Z, sets the pace of shoot meristem activity. Nature 569: 714-717. 
Whitewoods CD, Cammarata J, Venza ZN, Sang S, Crook AD, Aoyama T, Wang XY, Waller M, Kamisugi Y, Cuming AC, et al. 2020. CLAVATA Was a Genetic Novelty for the Morphological Innovation of 3D Growth in Land Plants. Current Biology 30: 2645-2648.

737 Wynn AN, Seaman AA, Jones AL, Franks RG. 2014. Novel functional roles for 738 PERIANTHIA and SEUSS during floral organ identity specification, floral meristem termination, and gynoecial development. Frontiers in Plant Science 5.

Xing S, Rosso MG, Zachgo S. 2005. ROXY1, a member of the plant glutaredoxin family, is required for petal development in Arabidopsis thaliana. Development 132: 1555-1565.

Xing S, Zachgo S. 2008. ROXY1 and ROXY2, two Arabidopsis glutaredoxin genes, are required for anther development. The Plant Journal 53: 790-801. genes is defined by the LITTLE ZIPPER protein DTM in tomato. Communications Biology 2: 134.

\section{Yang F, Bui HT, Pautler M, Llaca V, Johnston R, Lee B, Kolbe A, Sakai H, Jackson D.} 2015. A Maize Glutaredoxin Gene, Abphyl2, Regulates Shoot Meristem Size and Phyllotaxy.

749 The Plant Cell 27: 121-131.

751 Spatiotemporally dynamic, cell-type-dependent premeiotic and meiotic phasiRNAs in maize

752 anthers. Proceedings of the National Academy of Sciences 112: 3146-3151. 